\title{
Learning from failure: persistence of aneurysms following pipeline embolization
}

\author{
Maksim Shapiro, MD, ${ }^{1,2}$ Tibor Becske, MD, ${ }^{3}$ and Peter K. Nelson, MD ${ }^{1,4}$ \\ Departments of ${ }^{1}$ Radiology, Bernard and Irene Schwartz Neurointerventional Radiology Section, ${ }^{2}$ Neurology, and ${ }^{4}$ Neurosurgery, \\ NYU School of Medicine, New York; and ${ }^{3}$ Department of Neurology, Rochester General Hospital, Rochester Regional Health \\ System, Rochester, New York
}

OBJECTIVE A detailed analysis was performed of anterior circulation aneurysms treated with a Pipeline Embolization Device (PED) that did not progress to complete occlusion by 1 -year follow-up. Angiography was performed with the purpose of identifying specific factors potentially responsible for these failed outcomes.

METHODS From among the first 100 patients with anterior circulation aneurysms, 92 underwent 1-year follow-up angiography and were individually studied through review of their pre- and postembolization studies.

RESULTS Nineteen aneurysms (21\%) remained unoccluded at 12 months. Independent predictors of treatment failure, identified by logistic regression analysis, were found to be fusiform aneurysm morphology, decreasing dome-to-neck ratio, and the presence of a preexisting laser-cut stent. Further examination of individual cases identified several common mechanisms-device malapposition, inadequate coverage of the aneurysm neck with persistent exchange across the device, and the incorporation of a branch vessel into the aneurysm fundus-potentially contributing to failed treatment in these settings.

CONCLUSIONS Attention to specific features of the aneurysm and device construct can frequently identify cases predisposed to treatment failure and suggest strategies to maximize favorable outcomes.

http://thejns.org/doi/abs/10.3171/2015.12.JNS152065

KEY WORDS Pipeline Embolization Device; aneurysm; brain; vascular disorders

$\mathrm{T}$ He Pipeline Embolization Device (PED) has been in use since 2007. In 2011, the PED received FDA clearance for use in the US, based on results of the Pipeline for Uncoilable or Failed Aneurysms (PUFS) ${ }^{1}$ trial, reporting $86.8 \%$ aneurysm occlusion at 12 -month follow-up angiography among a cohort of large, wideneck aneurysms of the internal carotid artery (ICA). The worldwide experience has since been characterized by device utilization in a more varied aneurysm population, ${ }^{7}$ and by the overall trend toward limiting the number of implanted devices per case, compared with the 3-device median per aneurysm in the PUFS trial. An occlusion rate of $76 \%$ at 6-month follow-up was calculated in a recent meta-analysis of the flow diversion literature ${ }^{3}$ that includ- ed treatment of smaller aneurysms than those reported in the PUFS trial.

The processes underlying successful aneurysm closure with the PED and other minimally porous endoluminal devices (MPEDs) have been discussed at length elsewhere. ${ }^{6}$ Briefly, implantation of an MPED or multidevice construct across the aneurysm neck immediately modifies flow within the aneurysm and reconstructed arterial lumen, together referred to as flow diversion. The resulting intraaneurysmal stasis leads to thrombosis within the aneurysmal sac in most cases. Although frequently rendering the aneurysm angiographically occult, the initial thrombosis does not immediately produce a cure nor offer complete protection against delayed rupture or expanding mass effect, but

ABBREVIATIONS ICA = internal carotid artery; MPED = minimally porous endoluminal device; $\mathrm{PCOA}=$ posterior communicating artery; $\mathrm{PED}=\mathrm{Pipeline} \mathrm{Embolization}$ Device; PRU = platelet reactivity unit; PUFS = Pipeline for Uncoilable or Failed Aneurysms; ROC = receiver operating characteristic.

SUBMITTED September 1, 2015. ACCEPTED December 11, 2015.

INCLUDE WHEN CITING Published online May 6, 2016; DOI: 10.3171/2015.12.JNS152065. 
likely requires neointimal overgrowth of the construct to fully exclude the aneurysm from the circulation. ${ }^{6}$

This broad outline suggests conditions that must be met to achieve a cure, and possible factors that could interfere with it. This study focused on identifying the factors likely to produce such suboptimal outcomes.

\section{Methods}

The authors performed a retrospective review of a prospectively maintained institutional database of consecutive patients treated with the PED, which was approved by the Institutional Review Board. Posterior circulation and extracranial PED cases were excluded. All aneurysms were unruptured. Adjunctive coil embolization was performed in 9 cases, as deemed appropriate on an individual basis. All patients were pretreated with aspirin and clopidogrel, using several loading protocols congruent with overall medical management. The VerifyNow Assay (Accumetrics) was used in all cases to monitor platelet reactivity and adjust dosage for a target value of less than 220 P2Y12 platelet reactivity units (PRUs). Nonresponders were given additional clopidogrel until target P2Y12 values were achieved. Hyper-responders, defined as PRU $<20$, were managed by reducing clopidogrel dosage in proportion to the degree of response. Dual antiplatelet therapy was continued for 6 months after the procedure; clopidogrel was either stopped or reduced after 6 months, depending on individual factors, whereas aspirin was continued indefinitely.

From among our first 100 patients with anterior circulation aneurysms, 92 underwent 1-year follow-up angiography. Procedural and follow-up angiograms were individ- ually studied by all authors. The presumed cause or causes for nonocclusion were adjudicated and a consensus opinion established. Associations between 12-month occlusion and presence of a preexisting stent and fusiform aneurysm morphology were assessed with a 2-tailed Fisher exact test. Contiguous and multiple categorical variable associations, including dome size, neck size, dome-to-neck ratio, number of PEDs used, and aneurysm location, underwent receiver operating characteristic (ROC) curve analysis. Associations thus found to be significant were then subjected to a logistic regression analysis to identify factors independently predictive of treatment failure.

\section{Results}

Nineteen cases of incomplete target aneurysm occlusion $(21 \%)$, listed in Table 1 by aneurysm location, ${ }^{12}$ were found among 92 of our first 100 consecutive patients with large and giant ICA aneurysms with available 1-year follow-up. Preexisting stents, from prior stent-assisted coiling, were significantly and independently associated with 12-month treatment failure based on the 2-tailed Fisher exact test $(p=0.03)$ and by logistic regression analysis $(p$ $=0.02$ ). Increasing aneurysm complexity, as reflected by saccular lesions with lower dome-to-neck ratios and fusiform aneurysm morphologies (circumferentially involving a vascular segment), was significantly and independently associated with treatment failure (fusiform morphology: $\mathrm{p}$ $=0.03$, Fisher exact test, and $\mathrm{p}=0.02$, logistic regression; dome-to-neck ratio: $\mathrm{p}=0.004$, $\mathrm{ROC}$, and $\mathrm{p}=0.007$, logistic regression). Neither aneurysm fundus size nor neck size alone achieved statistical significance. A larger num-

TABLE 1. Summary of 12-month nonocclusion cases

\begin{tabular}{|c|c|c|c|c|c|c|c|c|}
\hline $\begin{array}{l}\text { Case } \\
\text { No. }\end{array}$ & $\begin{array}{l}\text { Aneurysm } \\
\text { Location }\end{array}$ & $\begin{array}{l}\text { Age } \\
\text { (yrs) }\end{array}$ & $\begin{array}{l}\text { Previous } \\
\text { Treatment }\end{array}$ & $\begin{array}{l}\text { Dome } \\
(\mathrm{mm})\end{array}$ & $\begin{array}{l}\text { Neck } \\
(\mathrm{mm})\end{array}$ & $\begin{array}{c}\text { Dome/ } \\
\text { Neck Ratio }\end{array}$ & $\begin{array}{l}\text { No. of } \\
\text { PEDs }\end{array}$ & Proposed Mechanism of Aneurysm Persistence \\
\hline 1 & Cavernous & 69 & & 12.2 & 4.9 & 2.5 & 3 & Distal landing zone endoleak \\
\hline 2 & Cavernous & 68 & Coil, stent & 16.2 & 13.5 & 1.2 & 5 & Preexisting Neuroform stent w/ secondary endoleak formation \\
\hline 3 & Cavernous & 76 & & 17.8 & 13.9 & 1.3 & 3 & Persistent exchange at curve convexity \\
\hline 4 & Cavernous & 66 & & 25 & 7.7 & 3.2 & 3 & Persistent exchange at curve convexity \\
\hline 5 & Cavernous & 66 & & 26.4 & 12 & 2.2 & 4 & Persistent exchange at curve convexity \\
\hline 6 & Cavernous & 37 & & 27.5 & 18 & 1.5 & 3 & Persistent exchange at curve convexity \\
\hline 7 & Paraophthalmic & 73 & & 3.1 & 2.7 & 1.1 & 2 & Persistent exchange \\
\hline 8 & Paraophthalmic & 46 & & 7 & 5.6 & 1.3 & 3 & Ophthalmic artery runoff maintains aneurysm inflow \\
\hline 9 & Paraophthalmic & 57 & & 10.2 & 7 & 1.5 & 3 & Ophthalmic artery runoff maintains aneurysm inflow \\
\hline 10 & Paraophthalmic & 75 & & 11.5 & 6.6 & 1.7 & 3 & Persistent exchange \\
\hline 11 & Paraophthalmic & 73 & & 12.6 & 8.5 & 1.5 & 4 & Ophthalmic artery runoff maintains aneurysm inflow \\
\hline 12 & Paraophthalmic & 72 & & 20.3 & 9.4 & 2.2 & 3 & Ophthalmic artery runoff maintains aneurysm inflow \\
\hline 13 & Paraophthalmic & 65 & Coil, stent & 21.6 & 9.2 & 2.3 & 3 & Preexisting Neuroform stent w/ secondary endoleak formation \\
\hline 14 & PCoA & 73 & & 8.4 & 6.1 & 1.4 & 2 & PCoA artery runoff maintains aneurysm inflow \\
\hline 15 & PCoA & 65 & Coil, stent & 10.9 & 5.6 & 1.9 & 2 & $\begin{array}{l}\text { Neuroform across PCoA aneurysm; stent \& PCoA keep aneurysm } \\
\text { open }\end{array}$ \\
\hline 16 & PCoA & 82 & & 13.6 & 6.8 & 2.0 & 4 & PCoA artery runoff maintains aneurysm inflow \\
\hline 17 & Petrous & 23 & & 40.9 & 40.9 & 1.0 & 10 & Giant petrous aneurysm; several foci of persistent exchange \\
\hline 18 & Petrous & 40 & & 50 & 50 & 1.0 & 7 & Giant petrous aneurysm; several foci of persistent exchange \\
\hline 19 & Petrous & 50 & & 60 & 60 & 1.0 & 8 & Giant petrous aneurysm; several foci of persistent exchange \\
\hline
\end{tabular}


ber of PEDs was found to be predictive of failure by ROC analysis but not by logistic regression, consistent with its confounding role as a marker for increasing aneurysm complexity.

Further evaluation of individual cases of treatment failure identified several radiographic findings common to failed treatment: 1) inadequate aneurysm coverage, leading to persistent exchange between the parent vessel and the aneurysm across the implant; 2) impaired implantation of the PED in the presence of a formerly placed laser-cut stent; 3) incorporation of branch vessels into the aneurysm fundus; and 4) evolving endoleak, arising as a consequence of primary maldeployment of the PED.

\section{Discussion}

This study demonstrates a relationship between the anatomical complexity of the aneurysm-as reflected by aneurysms with low dome-to-neck ratios and fusiform morphologies-and MPED treatment failure. Although the concept of dome-to-neck ratio is more commonly invoked as a predictor of coiling treatment failure, our re- sults suggest its validity for endoluminal treatment analysis as well. Several mechanisms may account for observed suboptimal outcomes.

\section{Inadequate Aneurysm Coverage}

Morphologically, larger and more complex aneurysms necessitate the creation of longer device constructs, with increasing potential for insufficient metal coverage or incomplete/deficient endothelialization. The extent of metal coverage for any given PED implant varies substantially as a function of device size relative to the size of the artery into which it is implanted; the degree of vessel curvature that must be accommodated by the device; number of devices used; and factors related to technique, such as degree of load or traction used in deployment of the device. ${ }^{2,8,13,14}$ Oversizing the PED typically leads to substantial decreases in metal coverage, ${ }^{14}$ especially along curvatures. ${ }^{16,17}$ Treatment failure related to undercoverage typically manifests as broad areas of persistent exchange across the PED construct (Figs. 1 and 2). However, even where multidevice constructs are used, the explicit cov-
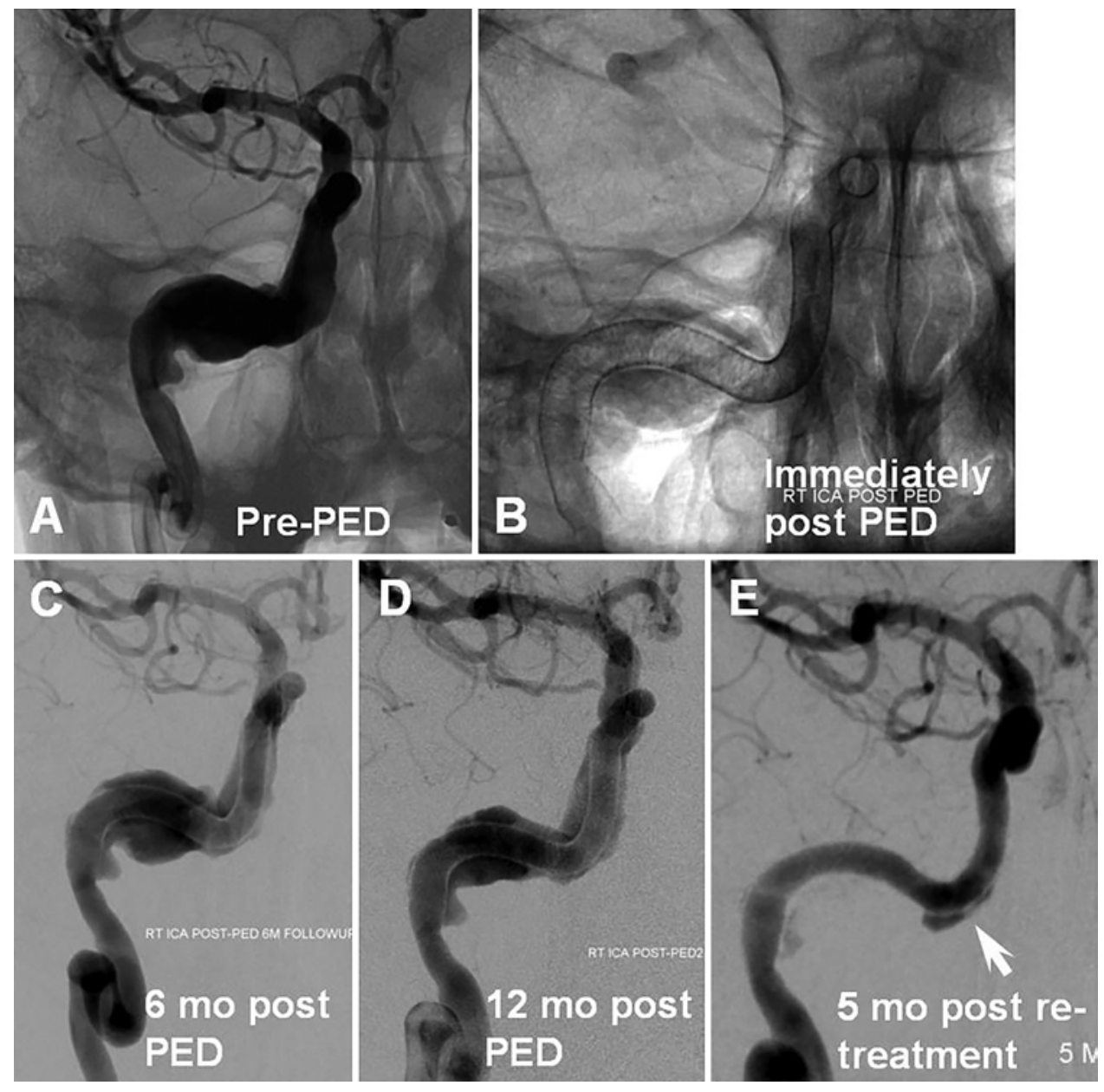

FIG. 1. Case 18. A and B: Giant petrous segment aneurysm, initially treated with 7 PEDs. C: The aneurysm is widely patent 6 months postembolization due to persistent exchange across the device mesh. D: The disposition is unchanged at 12 months postembolization, and 5 additional PEDs are added. E: Five months after the second embolization, several foci of persistent exchange remain, notably along the outer curvature of the distal petrous segment (arrow). 

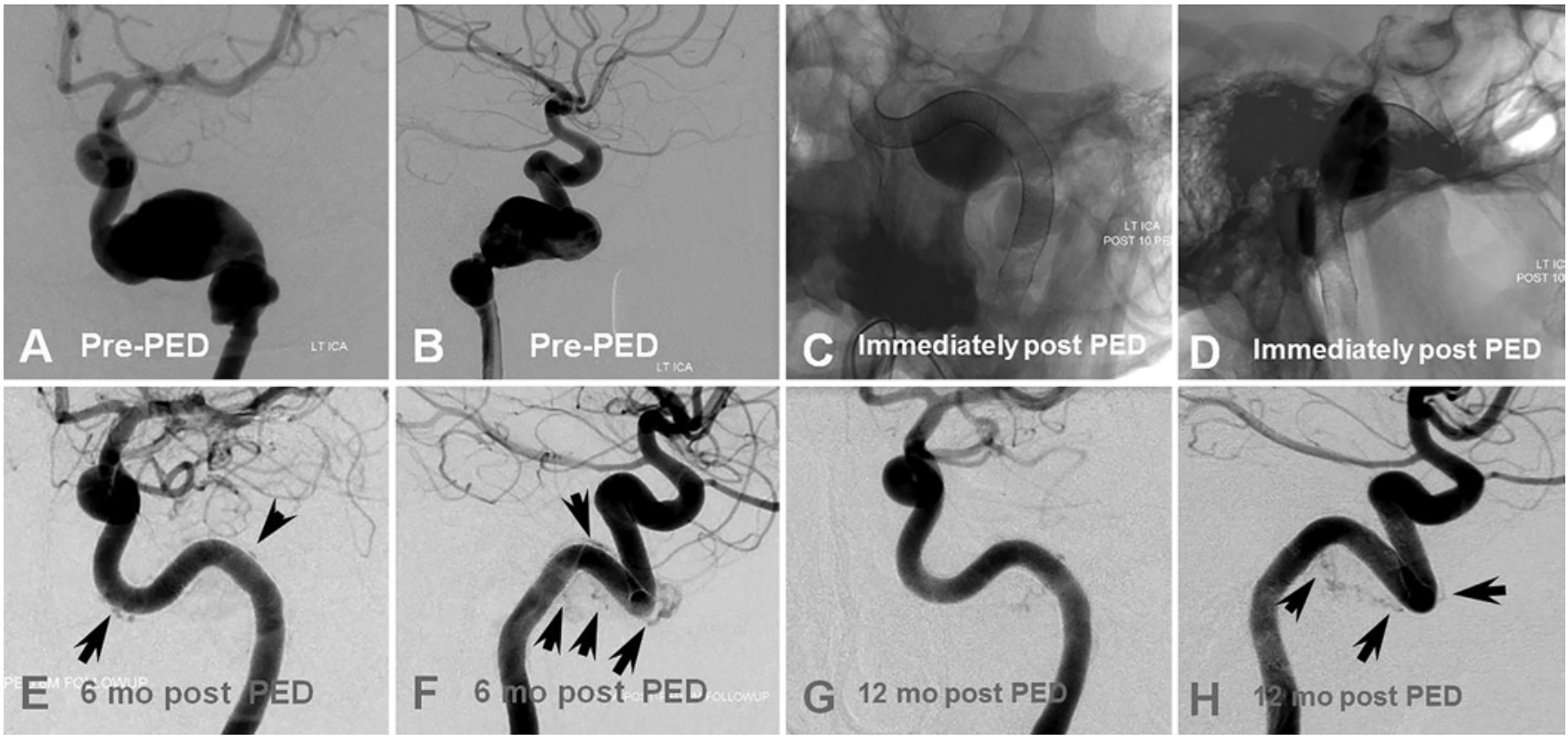

FIG. 2. Case 17. A-D: Giant cervical and petrous segment aneurysm, treated with 10 overlapping PEDs. E and F: At 6 months, multiple discrete foci of residual inflow through nonendothelialized areas of the construct remain. Notice location of the largest focus (arrows) at the apex of curve convexity, where metal coverage is expected to be relatively diminished. G and H: At 12 months posttreatment, continued endothelialization has reduced the number of inflow zones, with several persisting. Arrows $(\mathrm{H})$ indicate areas of continued inflow/outflow.

erage of the aneurysm neck may remain uneven due to variation in the exact overlay of braids upon each other (Fig. 3E). ${ }^{13}$ This factor is most pronounced in fusiform and extreme complex-neck aneurysms, where effective reconstruction can be aided by using a sufficient number of overlapping devices, consistent with the methodology validated in the PUFS trial. ${ }^{1}$ Nevertheless, persistent exchange (aneurysm inflow) across multidevice constructs can still be observed in refractory cases due to foci of minimized coverage, which may occur with periodic-
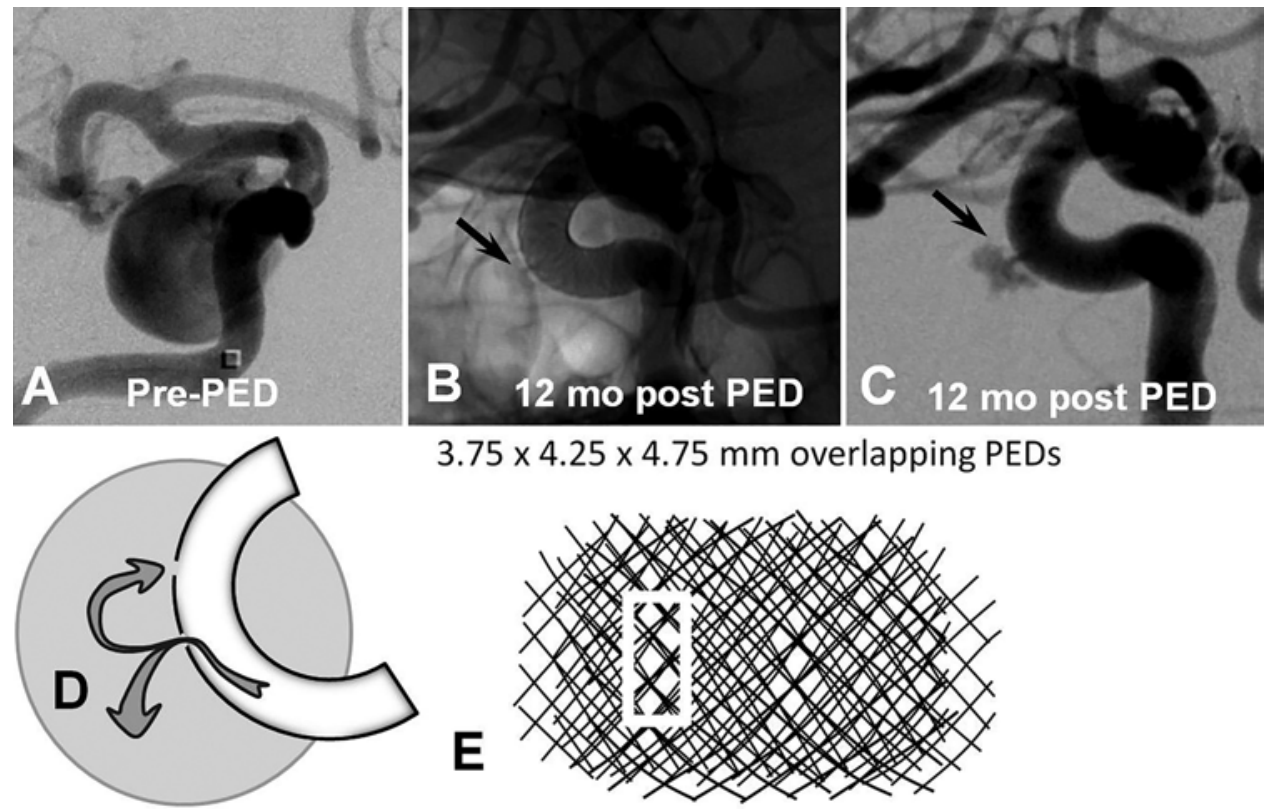

\section{$3.75 \times 4.25 \times 4.75 \mathrm{~mm}$ overlapping PEDs}

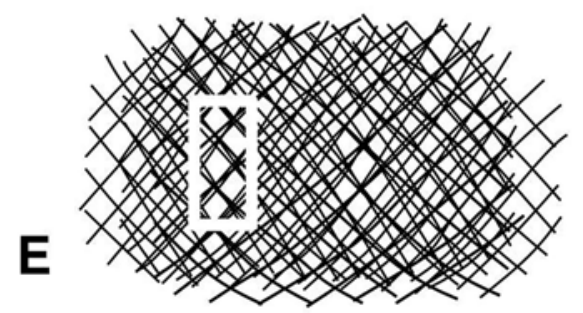

FIG. 3. Case 3. A-E: Focal contrast inflow along a segment of PED construct convexity (arrows, B and C), where metal coverage is expected to be particularly low. D: Schematic illustration of this mechanism. Outflow from the aneurysm can be directed back through the stent construct or, in case of cavernous aneurysms, into a dural branch such as the inferolateral or meningohypophyseal trunks. E: Three-stent overlap segmentation image, demonstrating the presence of several isolated cells with low focal coverage despite high overall coverage values, as demonstrated in a recent publication by Shapiro et al. ${ }^{13}$ 
ity along constructs of overlapping devices (Fig. 3E). We identified 4 cases where persistent aneurysm opacification proceeds through submillimeter holes in multistent constructs, typically located along curvature convexities (Fig. 3). This phenomenon was observed in cases with an otherwise aggressive endothelial reaction, suggesting that local geometrical inhomogeneities in the overlapping devices rather than the assumed average construct coverage was a factor in maintaining aneurysm patency. All unoccluded fusiform aneurysms (Cases 6, 17-19) in our series underwent retreatment with additional PEDs; 3 have since gone on to complete closure, with follow-up pending in the remaining case.

\section{Preexisting Stents}

Reduced efficacy of the PED in the face of preexisting stents has been previously described. ${ }^{5,9}$ In this series, $3(75 \%)$ of 4 cases with preexisting stents remained unoccluded at 12 months (Table 1). Laser-cut stents, such as the Neuroform (Stryker) or Enterprise (Cordis), particularly when implanted along an acute vessel curve, often do not fully appose the parent artery wall, either as a consequence of the rigidity of their component crowns (in opencell devices), or as a result of partial folding and kinking of closed-cell devices. ${ }^{5}$ Navigating through the "true lumen" of these implants may be difficult, complicating subsequent PED deployment and implantation within the parent vessel. MPEDs deployed within these stents frequently do not fully appose the wall of the target vessel, unmasking the preexisting nonapposition of the indwelling stent, and potentially leading to establishment of endoleaks, or contributing to inadequate intimal overgrowth of the newly deployed MPED (Fig. 4). Extending the MPED landing zone distal and proximal to the indwelling device (to allow implantation of the device in an intimal competent vascular segment and reduce endoleakage) is likely to maximize efficacy, although it remains problematic in our experience.

\section{Branch Vessel Runoff}

Continued patency of normal branch vessels following their coverage by MPEDs is dependent on several factors, including the degree of metal coverage, the thrombogenic disposition of the patient, the degree of individual intimal reactivity, and interrelated factors affecting the flow through the jailed ostium (such as the size of the vessel and its vascular territory, the resistance to flow within the branch vasculature, and the availability of collateral support). When a branch vessel is incorporated into the target aneurysm, its runoff can potentially contribute to persistent filling of the aneurysm by the very same physiological processes theoretically responsible for the preservation of jailed branch vessels and perforators arising from normal segments.

Four ophthalmic (Table 1, Cases 8, 9, 11, and 12; Fig. 5) and 3 posterior communicating segment aneurysm cases (Cases 14-16) were identified, as well as one anterior choroidal aneurysm described elsewhere. ${ }^{11}$ Even under conditions in which a neighboring branch does not directly arise from the aneurysm, an analogous anatomical dis-
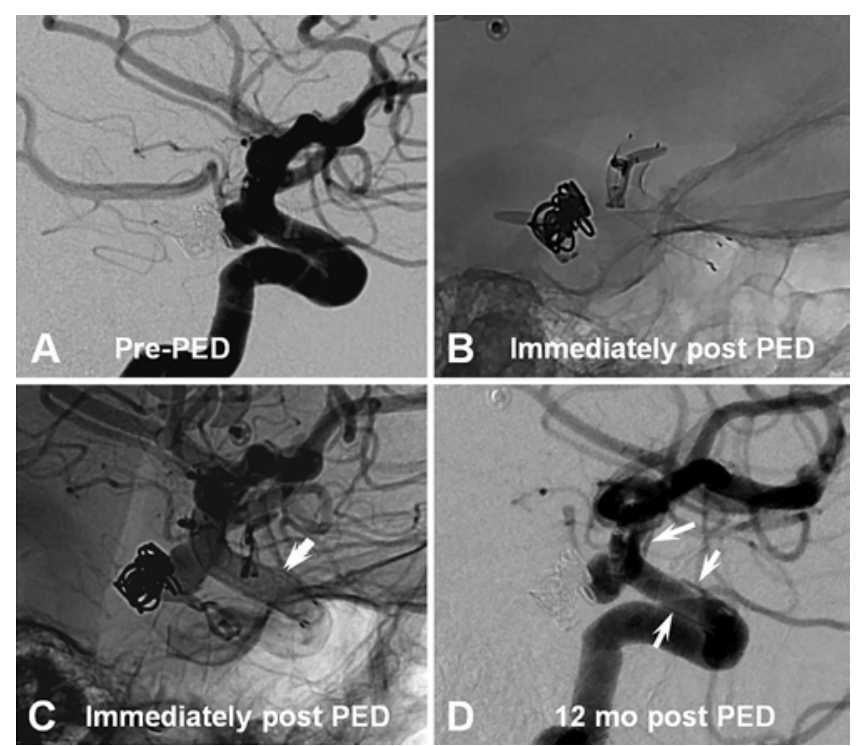

FIG. 4. Case 15. Aneurysm occlusion failure in the setting of an indwelling Neuroform stent. A: Recurrent, previously ruptured PCoA aneurysm is treated by placement of additional coils and a Neuroform device, followed later by another recurrence. B: The PED is placed within the Neuroform stent. C and D: Persistence of aneurysm. The Neuroform stent prevents apposition of the PED to the vessel wall, resulting in multiple areas of endoleakage (arrows).

position may be artificially created by deployment of an undersized device in the aneurysmal segment, bringing the ostium of a nearby branch and the aneurysm neck into a common compartment external to the construct (Case 11; Fig. 6). In this construct, the branch artery serves as a potential source of outflow for the aneurysm compartment external to the newly created construct (Fig. 6C). Selection of larger diameter devices to avoid this type of externalized aneurysm-adjacent branch vessel compartment must be balanced with goals of achieving adequate metal coverage and avoiding aberrant device implantation, more commonly noted with device oversizing. . $^{3,14}$

One may expect that final closure of such aneurysms would require concomitant occlusion of the associated branch, ${ }^{15}$ as we observed in cases in which the aneurysm closed spontaneously in a delayed fashion (Fig. 6D), or following retreatment of ophthalmic aneurysms with additional PEDs (Fig. 5E and F), as has been previously reported in multiple case reports of failed ophthalmic, ${ }^{4,10}$ anterior choroidal, and PCoA aneurysms. ${ }^{11,15}$

\section{Endoleak}

Both proximal and distal endoleaks can interfere with aneurysm occlusion. Although the classic endoleak is not present immediately after treatment due to the porosity of the MPED construct, endoleaks can evolve as an incompletely imbedded device becomes variably covered by neointimal overgrowth, permitting continued filling of the aneurysm through an aberrant channel that develops between the device and the vessel wall over the segment where the construct is unapposed. Thus, endoleakage implies inadequate device-to-wall apposition. We identified 1 distal landing zone endoleak (Case 1; Fig. 7) success- 


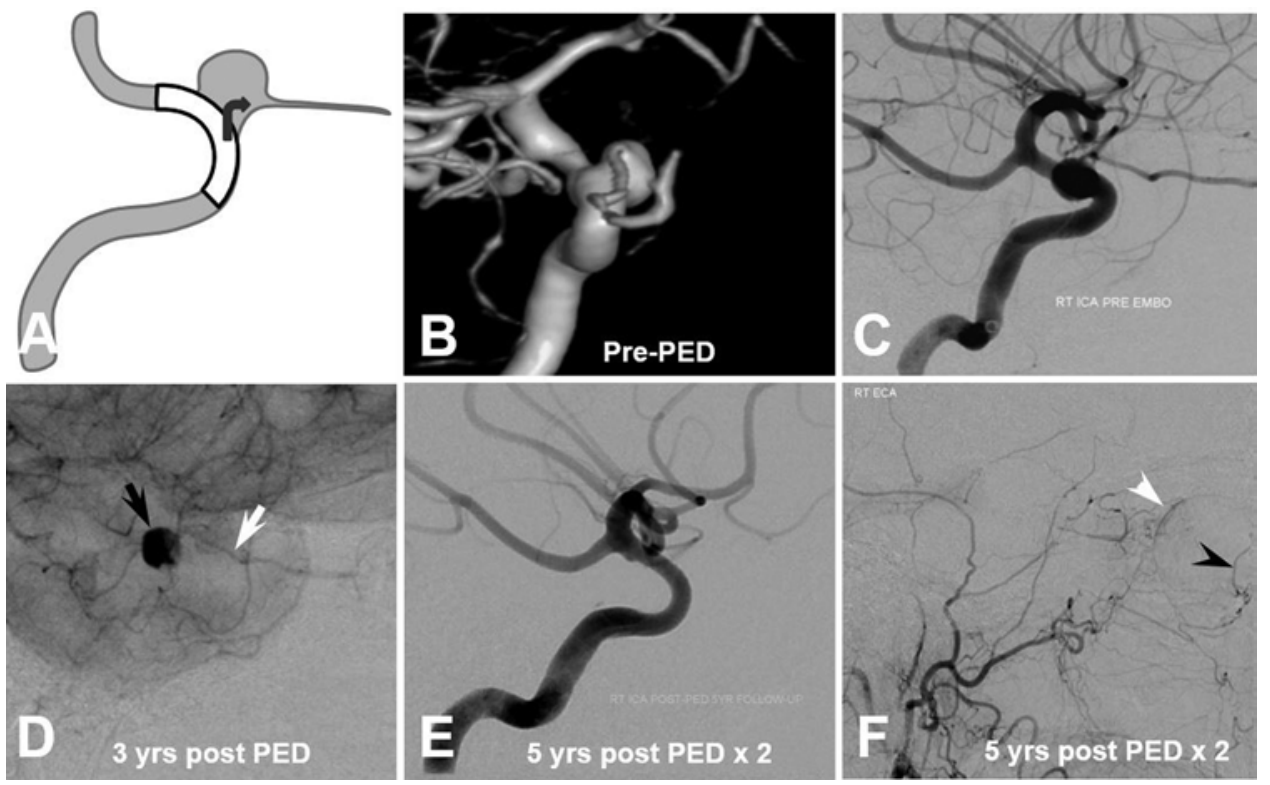

FIG. 5. Case 9. A-C: The dome of the aneurysm incorporates the ophthalmic artery. D: Three years following embolization, the ophthalmic artery (white arrow) constitutes the outflow of a persistently unoccluded aneurysm (black arrow). E: Five years after initial embolization, and following placement of 2 additional PEDs, the aneurysm is closed, with occlusion of the ophthalmic artery as a prerequisite. F: The ophthalmic artery fills from external carotid circulation, opacifying the choroid (white arrowhead) and the circle of the iris (black arrowhead), with no visual deficit.

fully closed by placing an additional PED across the distal landing zone.

Identification of deficient device-to-wall apposition can be aided by careful postdeployment biplane angiography in the unsubtracted mode, and DynaCT imaging evaluation, wherever appropriate. Deficiencies may be addressed using the J-wire technique, "bumping" the proximal edge with the delivery catheter, or balloon angioplasty. Established endoleaks can be successfully managed by implanting an additional PED across the leak.

\section{Study Limitations}

This study has limitations, which include its retrospective design and demonstrably subjective evaluation, with no core lab validation. Several potential critical factors that might influence occlusion, such as the degree of individual endothelialization propensity, could not be formally examined. Determination of aneurysm occlusion was based on angiographic rather than transaxial imaging, precluding the possibility of identifying an increasingly recognized subgroup of angiographically occluded yet persistently expanding (thrombosed) aneurysms. The explanation for this phenomenon is likely one of complete intraaneurysmal thrombosis (and therefore apparent angiographic occlusion) in the setting of incomplete endothelialization of the construct, potentially leading to continued aneurysm growth and possible recanalization.

\section{Conclusions}

Mechanisms for aneurysm nonocclusion can be frequently identified by examination of individual cases. Persistent filling of an aneurysm requires the presence of both inflow and outflow zones. Preexisting stents, complex and fusiform aneurysm morphology, branch vessel runoff, and technical shortcomings such as endoleaks are responsible for maintenance of such inflow and outflow foci. A priori consideration of these factors can help identify aneurysms

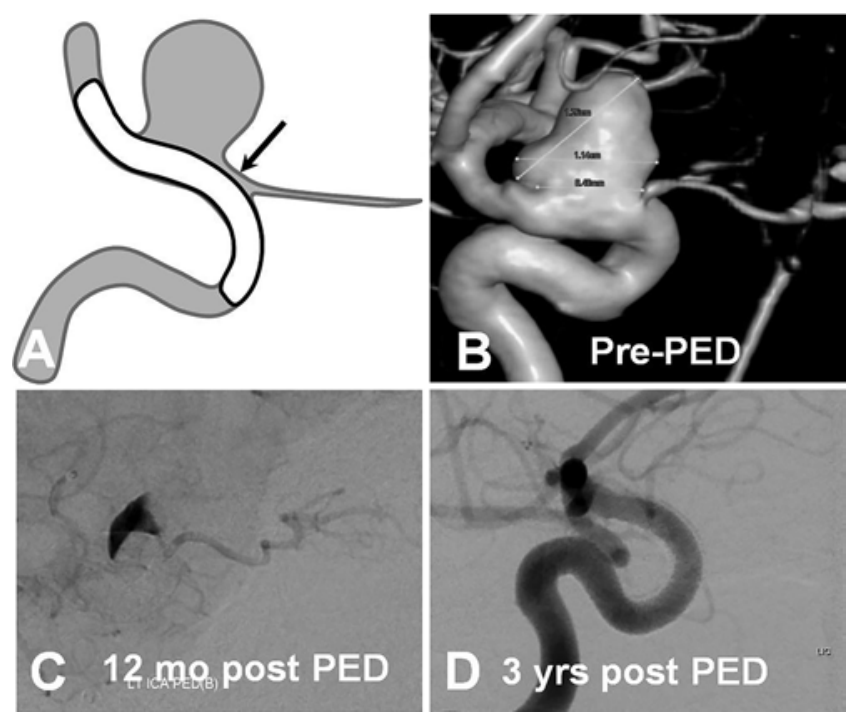

FIG. 6. Case 11. Secondary endoleak formation. A and B: The ophthalmic artery, initially separate from the aneurysm, is secondarily incorporated into the aneurysmal compartment by placement of an undersized device (arrow). C: At 12 months postreatment the ophthalmic artery now fills from the aneurysm, rather than through its natural ostium, in accordance with the mechanism shown in A. D: At 3 years, both the aneurysm and ophthalmic artery are closed. 

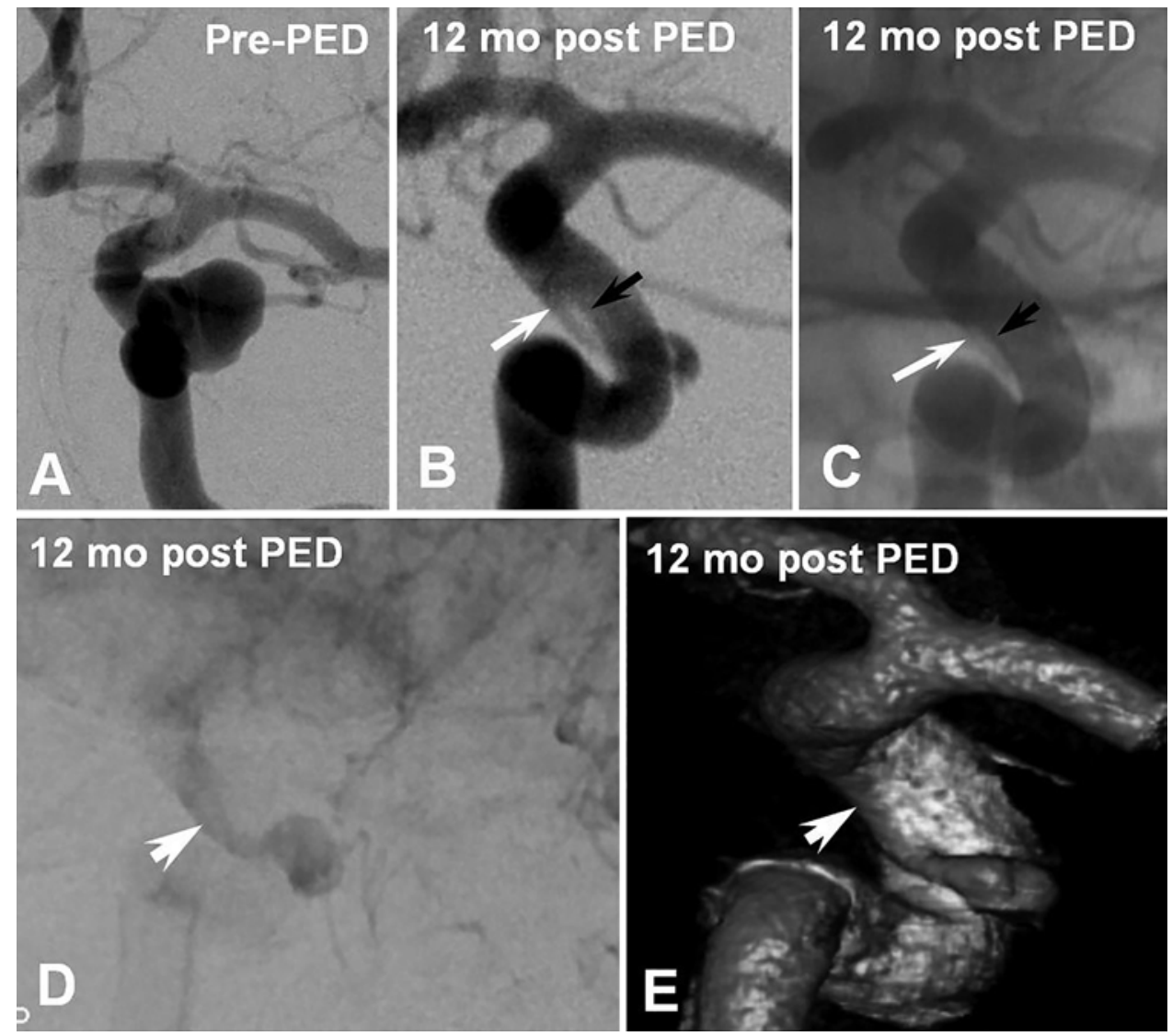

FIG. 7. Case 1. Paraophthalmic aneurysm (A). Distal endoleak due to nonapposition of the device (black arrows) and the distal landing zone (white arrows) seen in $\mathbf{B}$ and $\mathbf{C}$, establishing an inflow channel (arrowheads) seen in $\mathbf{D}$ and $\mathbf{E}$. The aneurysm was successfully closed by extending the PED construct into the distal hypophyseal segment.

at increased risk for nonocclusion and suggest strategies to address the situation.

\section{Acknowledgments} sis.

We thank James Babb, $\mathrm{PhD}$, for assistance with statistical analy-

\section{References}

1. Becske T, Kallmes DF, Saatci I, McDougall CG, Szikora I, Lanzino G, et al: Pipeline for uncoilable or failed aneurysms: results from a multicenter clinical trial. Radiology 267:858868, 2013

2. Bing F, Darsaut TE, Salazkin I, Makoyeva A, Gevry G, Raymond J: Stents and flow diverters in the treatment of aneurysms: device deformation in vivo may alter porosity and impact efficacy. Neuroradiology 55:85-92, 2013

3. Brinjikji W, Murad MH, Lanzino G, Cloft HJ, Kallmes DF: Endovascular treatment of intracranial aneurysms with flow diverters: a meta-analysis. Stroke 44:442-447, 2013

4. Chapot R, Mosimann P, Stauder M, Nordneyer H, Velasco A: Patency of a side branch as a factor to explain failure of flow diverters in ABC-WIN 2013. Val d'Isere, France: ABCWIN, 2013 (Abstract)

5. Ebrahimi N, Claus B, Lee CY, Biondi A, Benndorf G: Stent conformity in curved vascular models with simulated aneurysm necks using flat-panel CT: an in vitro study. AJNR Am J Neuroradiol 28:823-829, 2007
6. Fiorella D, Lylyk P, Szikora I, Kelly ME, Albuquerque FC, McDougall CG, et al: Curative cerebrovascular reconstruction with the Pipeline embolization device: the emergence of definitive endovascular therapy for intracranial aneurysms. J Neurointerv Surg 1:56-65, 2009

7. Kallmes DF, Hanel R, Lopes D, Boccardi E, Bonafé A, Cekirge $S$, et al: International retrospective study of the pipeline embolization device: a multicenter aneurysm treatment study. AJNR Am J Neuroradiol 36:108-115, 2015

8. Makoyeva A, Bing F, Darsaut TE, Salazkin I, Raymond $\mathrm{J}$ : The varying porosity of braided self-expanding stents and flow diverters: an experimental study. AJNR Am J Neuroradiol 34:596-602, 2013

9. Nelson PK, Lylyk P, Szikora I, Wetzel SG, Wanke I, Fiorella D: The pipeline embolization device for the intracranial treatment of aneurysms trial. AJNR Am J Neuroradiol 32:34-40, 2011

10. Puffer RC, Kallmes DF, Cloft HJ, Lanzino G: Patency of the ophthalmic artery after flow diversion treatment of paraclinoid aneurysms. J Neurosurg 116:892-896, 2012

11. Raz E, Shapiro M, Becske T, Zumofen DW, Tanweer O, Potts $\mathrm{MB}$, et al: Anterior choroidal artery patency and clinical follow-up after coverage with the Pipeline embolization device. AJNR Am J Neuroradiol 36:937-942, 2015

12. Shapiro M, Becske T, Riina HA, Raz E, Zumofen D, Jafar JJ, et al: Toward an endovascular internal carotid artery classification system. AJNR Am J Neuroradiol 35:230-236, 2014

13. Shapiro M, Raz E, Becske T, Nelson PK: Building 
multidevice pipeline constructs of favorable metal coverage: a practical guide. AJNR Am J Neuroradiol 35:1556-1561, 2014

14. Shapiro M, Raz E, Becske T, Nelson PK: Variable porosity of the Pipeline embolization device in straight and curved vessels: a guide for optimal deployment strategy. AJNR Am J Neuroradiol 35:727-733, 2014

15. Vedantam A, Rao VY, Shaltoni HM, Mawad ME: Incidence and clinical implications of carotid branch occlusion following treatment of internal carotid artery aneurysms with the pipeline embolization device. Neurosurgery 76:173-178, 2015

16. Wang K, Huang Q, Hong B, Li Z, Fang X, Liu J: Correlation of aneurysm occlusion with actual metal coverage at neck after implantation of flow-diverting stent in rabbit models. Neuroradiology 54:607-613, 2012

17. Wang K, Yuan S: Actual metal coverage at the neck is critical for flow-diverting stents in treating intracranial aneurysms.

AJNR Am J Neuroradiol 34:E31-E32, 2013

\section{Disclosures}

Drs. Nelson, Becske, and Shapiro are consultants and Pipeline proctors for Medtronic, manufacturer of the PED.

\section{Author Contributions}

Conception and design: all authors. Acquisition of data: all authors. Analysis and interpretation of data: all authors. Drafting the article: all authors. Critically revising the article: all authors. Reviewed submitted version of manuscript: Nelson.

\section{Correspondence}

Maksim Shapiro, Departments of Radiology and Neurology, Bernard and Irene Schwartz Neurointerventional Radiology Section, NYU School of Medicine, NYU Langone Medical Center, 660 First Ave., 7th Fl., New York, NY 10016. email: maksim.shapiro@nyumc.org. 